\title{
NUEVOS MATERIALES DE MACRANHINGA PARANENSIS NORIEGA (AVES, PELECANIFORMES, ANHINGIDAE) DEL MIOCENO DE LA PROVINCIA DE ENTRE RÍOS, ARGENTINA
}

\author{
JUAN MARCELO DIEDERLE, JORGE IGNACIO NORIEGA \\ Laboratorio de Paleontología de Vertebrados, Centro de Investigaciones Científicas y Transferencia de Tecnología \\ a la Producción (CICYTTP-CONICET), Dr. Matteri y España s/n, 3105, Diamante, Entre Ríos, Argentina. \\ juandiederle@yahoo.com.ar,cidnoriega@infoaire.com.ar \\ CAROLINA ACOSTA HOSPITALECHE \\ División Paleontología de Vertebrados, Museo de La Plata, Paseo del Bosque s/n, 1900, La Plata, Argentina. \\ acostacaro@fcnym.unlp.edu.ar
}

\begin{abstract}
NEW REMAINS OF MACRANHINGA PARANENSIS NORIEGA (AVES, PELECANIFORMES, ANHINGIDAE) FROM THE MIOCENE OF ENTRE RÍOS PROVINCE, ARGENTINA. Macranhinga paranensis Noriega, taxon described on the basis of tarsometatarsi, is known by dissociated remains of both girdles and limb bones coming from the "Conglomerado osífero" or "Mesopotamian" at Entre Ríos Province, Argentina (= base of the Ituzaingó Formation; late Miocene). In this contribution we report the first record of four isolated cervical vertebrae which are assigned to $M$. paranensis, coming from the Paraná Formation (middle-late Miocene) at La Ensenada stream (Diamante city, Entre Ríos Province) and from the "Conglomerado osífero" at La Toma Vieja fossiliferous locality (Paraná city, Entre Ríos Province). The systematic assignment of the new material is supported by different ratios calculated among the dimensions of vertebrae, pelvic girdles, and tarsometatarsi referred to $M$. paranensis, which were compared with the same proportions obtained in closed related taxa. The seventh vertebra presents several differences with that of Anhinga anhinga (Linnaeus) in the morphology of the foramen vertebrale and the facies articularis cranialis; the fifthteenth vertebra is very similar to that of $M$. ranzii Alvarenga \& Guilherme and has the zygapophysis cranialis proportionally broader and projected more posteriorly than in $A$. anhinga. Finally, the eighteenth vertebra has the foramen transversarium more rounded, the base of the zygapophysis caudalis with less ridged borders, and the torus dorsalis more prominent caudally than in A. anhinga. Thus, morphology, size, and metric proportions support the reference of the new materials to M. paranensis. The record of M. paranensis in the Paraná Formation (middle-late Miocene) extends the temporal range of this taxon.
\end{abstract}

Key words: Aves, Macranhinga paranensis, vertebrae, Miocene, Argentinian Mesopotamia.

RESUMO - A espécie Macranhinga paranensis Noriega, descrita a partir de tarsometatarsos, é também conhecida por restos desassociados de ambas as cinturas e membros, todos provenientes do "Conglomerado osífero" ou "Mesopotamiense" de Entre Ríos, Argentina (= base da Formação Ituzaingó; Mioceno tardio). O presente trabalho dá a conhecer quatro vértebras cervicais isoladas aqui atribuídas a M. paranensis, provenientes da Formação Paraná (Mioceno médio-tardio) no arroio La Ensenada (Diamante, Entre Ríos), e do "Conglomerado osífero" na localidade fossilífera Toma Vieja (Paraná, Entre Ríos). Para fundamentar sua classificação taxonômica, foram calculadas as relações métricas entre as vértebras, a pélvis e o tarsometatarso referidos a M. paranensis, as quais foram comparadas com as mesmas proporções em espécies filogeneticamente aparentadas. A sétima vértebra apresenta algumas diferenças morfológicas com a espécie atual Anhinga anhinga Linnaeus, principalmente no forame vertebral e nas facies articularis cranialis; a décima quinta vértebra é bastante similar a de M. ranzii Alvarenga $\&$ Guilherme e apresenta a zygapophysis cranialis proporcionalmente mais ampla e projetada mais posteriormente do que em $A$. anhinga (Linnaeus). Por último, na décima oitava vértebra, o foramen transversarium é mais arredondado, a base da zygapophysis caudalis com bordas menos agudas, e o torus dorsalis caudalmente mais proeminente do que em $A$. anhinga. A morfologia, assim como o tamanho e as proporções apóiam a assignação dos novos materiais a M. paranensis. O registro de M. paranensis na Formação Paraná (Mioceno medio-tardio) amplia o intervalo temporal da espécie.

Palavras-chave: aves, Macranhinga paranensis, vértebras, Mioceno, Mesopotâmia argentina.

\section{INTRODUCCIÓN}

Anhingidae es una familia de Pelecaniformes de amplia distribución mundial, distribuida predominantemente en regiones intertropicales de América, África, Asia y Oceanía. Las anhingas viven principalmente en sitios de agua dulce continentales, habitando incluso en ambientes marinos costeros, siempre que exista vegetación arbórea asociada (Orta, 1992). 
El registro más antiguo de la familia Anhingidae en América del Sur es Meganhinga chilensis Alvarenga, 1995, del Mioceno temprano de Chile (Charrier et al., 2007). El registro del Terciario en este subcontinente también comprende a otros tres géneros extintos y al género Anhinga Brisson, 1760, con las siguientes especies: A. cf. A. grandis Martin \& Mengel, 1975, en el Mioceno de Colombia (Rasmussen \& Kay, 1992; Rasmussen, 1997) y Mioceno de Brasil (Alvarenga \& Guilherme, 2003), $A$. fraileyi Campbell, 1996 y A. minuta Alvarenga \& Guilherme, 2003, ambas del Mioceno de Perú y Brasil, respectivamente; la segunda especie podría estar también presente en el Mioceno tardío de Argentina (Noriega \& Agnolin, 2008).

Los géneros monotípicos representados por las especies Meganhinga chilensis Alvarenga, 1995, Liptornis hesternus Ameghino, 1895 y Giganhinga kiyuensis Rinderknecht \& Noriega, 2002, se conocen del Mioceno temprano de Chile, Mioceno temprano tardío de Patagonia y del Plio-Pleistoceno de Uruguay, respectivamente. El género Giganhinga Rinderknecht \& Noriega, 2002 posiblemente se encuentre también representado en el Mioceno tardío de Entre Ríos (Areta et al., 2007).

Por último, el género Macranhinga Noriega, 1992 incluye dos especies: $M$. paranensis Noriega, 1992, exclusiva del Mioceno tardío de Argentina y $M$. ranzii Alvarenga \& Guilherme, 2003, hallada en el Mioceno tardío de Brasil y ciertamente también en Argentina (Noriega \& Agnolin, 2008). El género Macranhinga fue recientemente reportado en sedimentos del Mioceno temprano tardío de Patagonia (Cenizo \& Agnolin, 2010).

Macranhinga paranensis fue descrita a partir de tarsometatarsos aislados y completos (Noriega, 1992). Posteriormente se describieron más restos disociados del esqueleto apendicular, incluyendo huesos de las cinturas (Noriega, 1995, 2002; Noriega \& Piña, 2004; Noriega \& Agnolin, 2008). Comparada con las demás anhíngidas, M. paranensis es notablemente mayor que las especies actuales y fósiles del género Anhinga, menor que $M$. ranzii, y aún mucho más pequeña que G. kiyuensis. Sería un ave de aproximadamente $5,4 \mathrm{~kg}$, con predominio del vuelo batido sobre el planeo y con importantes adaptaciones asociadas al buceo (Noriega, 2001).

Todos los restos de M. paranensis provenían hasta el momento del "Conglomerado osífero" o "Mesopotamiense" (= base de la Formación Ituzaingó; Mioceno tardío; Figura 1) en la Provincia de Entre Ríos, Argentina (Figura 2). En la presente contribución se dan a conocer por primera vez elementos asignables a la columna vertebral de $M$. paranensis y uno de los cuales procede de niveles de la Formación Paraná (Mioceno medio-tardío; Figura 1).

\section{CONTEXTO GEOGRÁFICO Y ESTRATIGRÁFICO}

Durante el Mioceno, una parte del continente sudamericano se vio afectado por una transgresión marina conocida como "Mar Paranense o Entrerriense" (Del Río, 1991; Cione et al., 2000; Hernández et al., 2005), que ocupó una extensa área de Argentina, parte de Uruguay, sur de Brasil, sur de Bolivia y Paraguay.
Los afloramientos en el este de Entre Ríos corresponden a la sección cuspidal de la Formación Paraná (Bravard, 1858; Frenguelli, 1920; Aceñolaza, 1976, 2000; Aceñolaza \& Aceñolaza, 2000; Hernández et al., 2005) y se extienden desde el Departamento Victoria hasta las cercanías de la localidad de Hernandarias (Departamento La Paz). Esta unidad está constituida principalmente por arcillas grises y verdes oscuras, arcillas arenosas y limos arenosos, con delgadas intercalaciones de arenas finas, intermitentes bancos de arenas, con o sin estratificación, y zonas con carbonatos organógenos ricos en invertebrados y vertebrados marinos (Figura 1). La abundante presencia de restos fósiles permite establecer su edad entre el Mioceno medio (del Río, 1991) y el Mioceno tardío (Herbst \& Zabert, 1987; Cozzuol, 1993; Cione et al., 2000).

Durante el Mioceno más tardío, a medida que el "Mar Paranense" se retiraba, los abanicos fluviales se expandieron sobre el antiguo fondo marino dejando importantes depósitos arenosos (Aceñolaza, 2004) conocidos como Formación Ituzaingó (De Alba, 1953). Esta formación aflora en las barrancas del río Paraná y en muchos de sus tributarios de la margen izquierda, desde la ciudad homónima (norte de la Provincia de Corrientes) hasta los alrededores de la ciudad de Paraná (Herbst, 2000).

En la base de la Formación Ituzaingó aflora, de manera intermitente, entre las localidades entrerrianas de Hernandarias y Paraná, el relleno fluvial denominado "Conglomerado osífero" o "Mesopotamiense" por Frenguelli (1920) (Figura 1). El "Conglomerado osífero" presenta un espesor variable, con niveles de gravillas cuarzosas, clastos de arcilla y calcedonia, así como numerosos restos de vertebrados continentales y marinos, los últimos removidos de la Formación Paraná y posteriormente redepositados (Pascual \& Odreman Rivas, 1971).

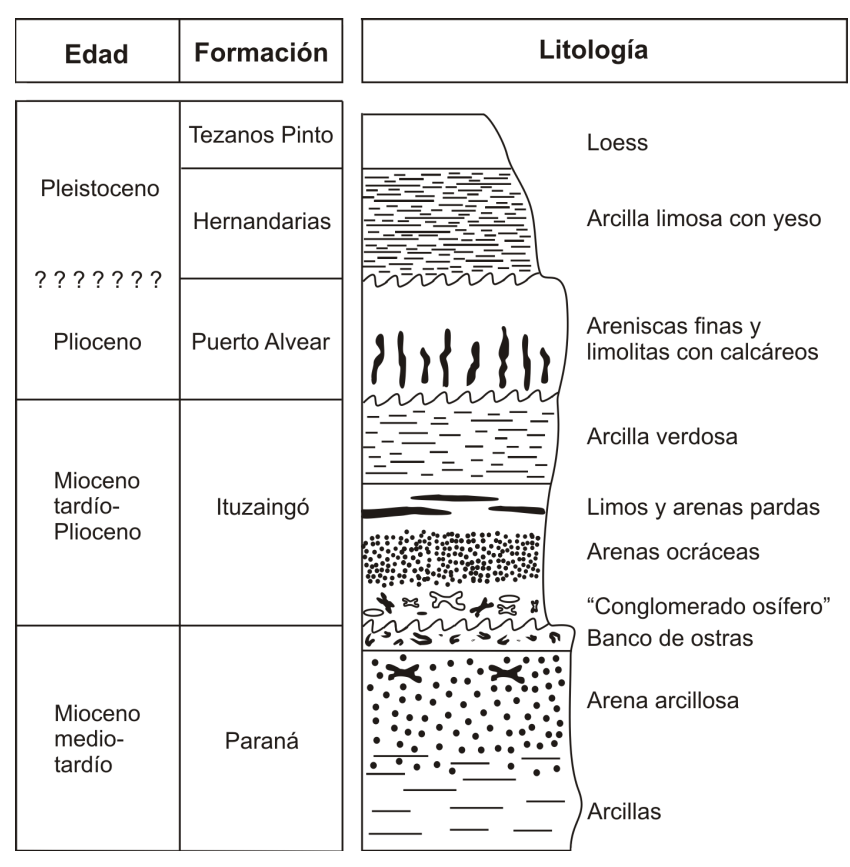

Figura 1. Perfil integrado del área de Paraná (modificado de Aceñolaza, 1976).

Figure 1. Integrated profile of the Paraná area (modified from Aceñolaza, 1976). 
Numerosos autores ubicaron a la edad de la Formación Ituzaingó entre el Mioceno y el Pleistoceno (e.g. Castellanos, 1965; Herbst, 1971; Bidegain, 1993). Actualmente, a partir de las relaciones estratigráficas existentes entre las diferentes unidades y por la información biocronológica aportada por los mamíferos, se propone para el "Conglomerado osífero" una edad Huayqueriense o Tortoniana (Mioceno tardío; Cione et al., 2000).

\section{MATERIAL Y MÉTODOS}

La determinaciones anatómicas se realizaron por medio de la comparación directa de los fósiles con las vértebras de $A$. anhinga (MACN 54828, MNHNP s $/ \mathrm{n}^{\circ}$ ). La nomenclatura osteológica fue tomada de Baumel et al. (1993). En las comparaciones biométricas, se asumió que las proporciones del esqueleto apendicular de $M$. paranensis eran similares a las de $A$. anhinga (Tabla 1). Se tomaron distintas medidas de las vértebras y de otros elementos esqueléticos previamente asignados a $M$. paranensis, M. ranzii, A. fraileyi y A. anhinga, a saber: $\mathbf{L C V}$, longitud del

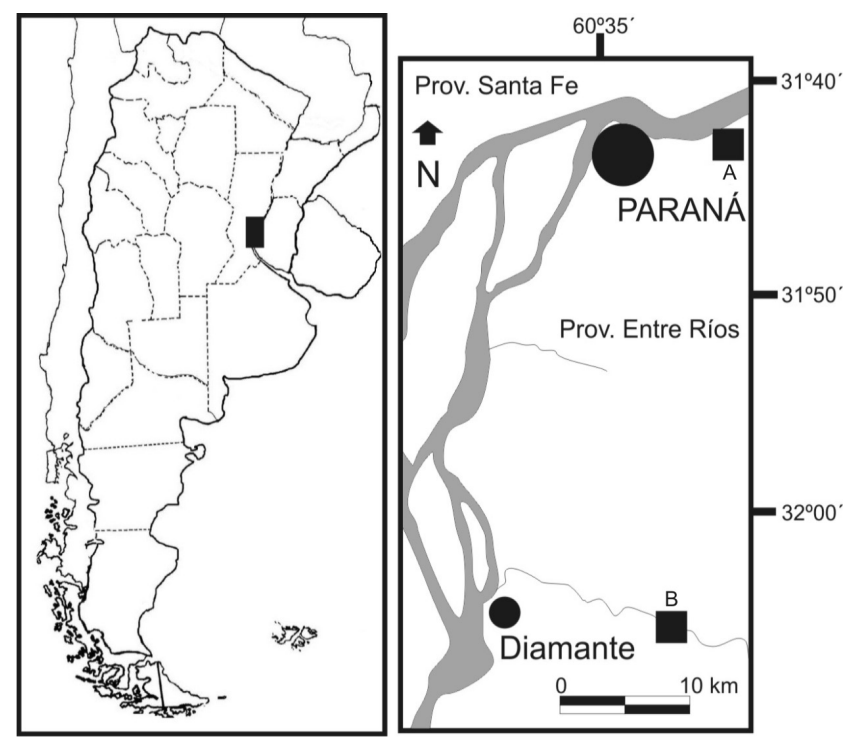

Figura 2. Mapa con las ubicaciones de las localidades fosilíferas: 1 , Toma Vieja, Paraná; 2, Arroyo La Ensenada, Diamante.

Figure 2. Map showing the fossiliferous localities. 1, Toma Vieja, Paraná; 2, Arroyo La Ensenada, Diamante. cuerpo vertebral; AFAC, ancho de la facies articularis cranialis; ATM, ancho de la tróclea del metatarsiano III; AAP, ancho entre los antitrocánteres de la pelvis.

Abreviaturas institucionales. CICYTTP, Centro de Investigaciones Científicas y de Transferencia de Tecnología a la Producción, Diamante, Entre Ríos, Argentina; MACN, Museo Argentino de Ciencias Naturales "Bernardino Rivadavia", Ciudad Autónoma de Buenos Aires, Buenos Aires, Argentina; MLP, Museo de La Plata, La Plata, Buenos Aires, Argentina; MNHN, Museo Nacional de Historia Natural, Asunción, Paraguay.

\section{SISTEMÁTICA PALEONTOLÓGICA}

Clase AVES Linnaeus, 1758

Orden PELECANIFORMES Sharpe, 1891

Familia ANHINGIDAE Ridgway, 1887

Género Macranhinga Noriega, 1992

Especie tipo. Macranhinga paranensis Noriega, 1992.

Macranhinga paranensis Noriega, 1992

(Figuras 3A-L)

Materiales referidos. CICYTTP-PV-A-2-243, fragmento de la séptima vértebra que preserva la porción mediocranial (Figuras 3A-C); CICYTTP-PV-A-2-242, vértebra decimoquinta con sus zygapophysis caudales y las foramina transversaria rotas (Figuras 3D-F); MLP 88-IX-20-18 y MACN 12750, vértebras decimoséptima y decimoctava casi completas (Figuras 3G-I y Figuras 3J-L, respectivamente).

Procedencia estratigráfica y geográfica. CICYTTPPV-A-2-243, Formación Paraná (Mioceno medio-tardío), localidad fosilífera arroyo La Ensenada, Departamento Diamante, Provincia de Entre Ríos, Argentina. CICYTTP-PV-A-2-242, MLP 88-IX-20-18 y MACN 12750, "Conglomerado osífero" o "Mesopotamiense" (Mioceno tardío), base de la Formación Ituzaingó, localidad fosilífera Toma Vieja, Departamento Paraná, Provincia de Entre Ríos, Argentina (Figura 2).

Descripción y comparaciones. Las vértebras son asignadas a la familia Anhingidae y diferenciadas de las de Phalacrocoracidae por los siguientes caracteres: processus ventralis proyectado caudalmente, foramen transversarium proporcionalmente

Tabla 1. Medidas (mm) y proporciones de los ejemplares estudiados. Abreviaturas: veja Material y Métodos.

Table 1. Measurements $(\mathrm{mm})$ and proportions of studied specimens. Abbreviations: see Material and Methods.

\begin{tabular}{|c|c|c|c|c|}
\hline & M. paranensis & A. anhinga & M. ranzii & A. fraileyi \\
\hline \multicolumn{5}{|l|}{ Vértebra 7} \\
\hline AFAC/AAP & 7,8/43,9: 0,177 & $5,9 / 32,7: 0,180$ & - & - \\
\hline AFAC/ATM & $7,8 / 10,6: 0,735$ & $5,9 / 5,2: 1,134$ & - & - \\
\hline \multicolumn{5}{|l|}{ Vértebra 15} \\
\hline LCV/AAP & $21,45 / 43,9: 0,488$ & $16,5 / 32,7: 0,504$ & - & - \\
\hline LCV/ATM & $21,45 / 10,6: 2,023$ & $16,5 / 5,2: 3,173$ & $29 / 13,4: 2,164$ & - \\
\hline \multicolumn{5}{|l|}{ Vértebra 18} \\
\hline LCV/AAP & $20,4 / 43,7: 0,466$ & $14,3 / 32,7: 0,437$ & - & - \\
\hline ATM & $20,4 / 10,6: 1,924$ & $14,3 / 5,2: 2,750$ & - & $16,6 / 8: 2,075$ \\
\hline
\end{tabular}


más reducido, tuberositas ligamenti collateralis menos cóncava, corpus más largo que ancho, sulcus caroticus más cóncavo y con crestas laterales más marcadas. A excepción de CICYTTP-PV-A-2-243, las vértebras estudiadas presentan un conspicuo reborde sobre la cara lateral del cuerpo y rugosidades superficiales, propias de una especie más robusta y con inserciones musculares más fuertes que las anhingas actuales. La vértebra séptima (CICYTTP-PV-A-2-243; Figuras $3 \mathrm{~A}-\mathrm{C}$ ) es un $32 \%$ más ancha que la de $A$. anhinga. El sulcus caroticus es profundo, bien definido y carente de crestas laterales, como en $A$. anhinga. La facies articularis cranialis es levemente más amplia y el margen dorsal del foramen vertebralis presenta un surco longitudinal más marcado que en $A$. anhinga. Las relaciones entre la AFAC y el AAP de $A$. anhinga son muy similares a las calculadas para $M$. paranensis. Por otra parte, la relación entre la AFAC y el ATM es menor en la vértebra estudiada que en $A$. anhinga
(Tabla 1). La vértebra decimoquinta (CICYTTP-PV-A-2-242; Figuras 3D-F) es un 30\% mayor que la correspondiente en $A$. anhinga y un $26 \%$ menor que la de $M$. ranzii. Su morfología general recuerda a $M$. ranzii (Alvarenga \& Guilherme, 2003: fig. 5A) y presenta un escaso desarrollo del processus spinosus, como en $A$. anhinga. Presenta la zygapophysis cranialis proporcionalmente más amplia y proyectada más posteriormente que en $A$. anhinga. Las relaciones entre el LCV y el AAP son muy similares a las de $A$. anhinga; en tanto que la proporción que vincula el LCV con el ATM es menor que en $A$. anhinga. Esta última proporción es similar a la observada en $M$. ranzii (Tabla 1 ). La vértebra decimoséptima (MLP 88-IX-20-18; Figuras 3G-I) posee un mayor desarrollo del processus ventralis respecto al de la decimoctava, lo que permite su posicionamiento en la columna vertebral. La vértebra decimoctava (MACN 12750; Figuras $3 \mathrm{~J}-\mathrm{L}$ ) es un $43 \%$ mayor que la de $A$. anhinga y un

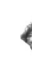

A

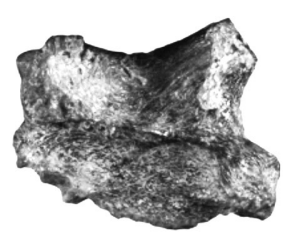

D
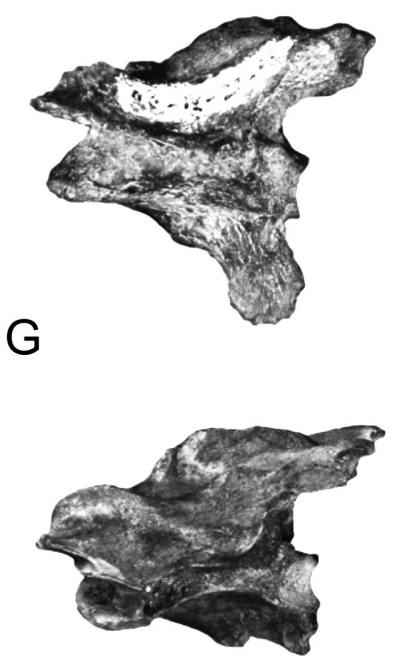

J

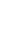

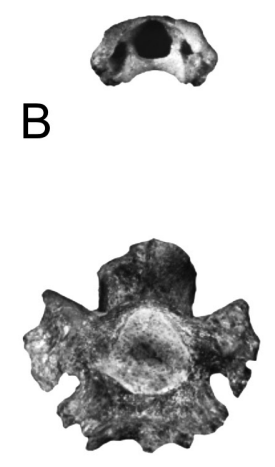

E
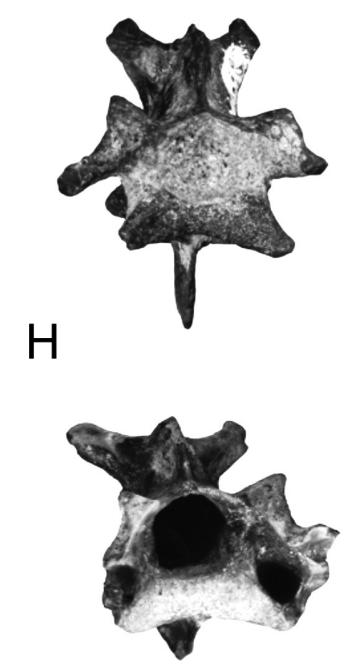

K
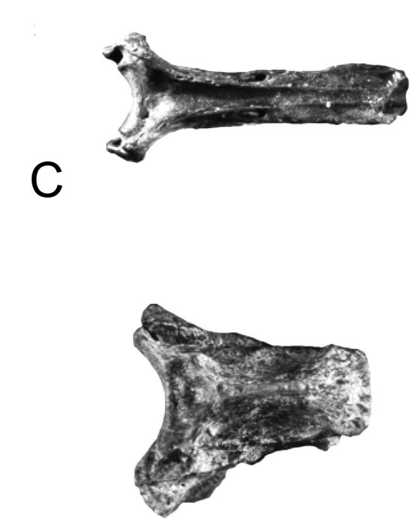

F

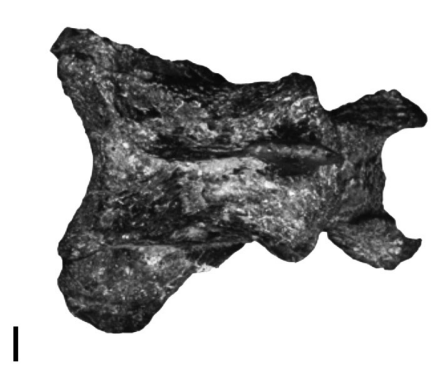

L

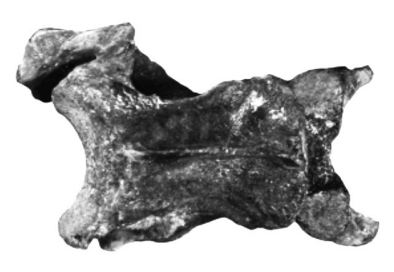

Figura 3. Macranhinga paranensis. CICYTTP-PV-A-2-243, séptima vértebra en vistas lateral (A), craneal (B) y ventral (C); CICYTTP-PV-A-2-242, decimoquinta vértebra en vistas lateral (D), craneal $(\mathbf{E})$ y ventral $(\mathbf{F})$; MLP 88-IX-20-18, decimoséptima vértebra en vistas lateral (G), craneal (H) y ventral (I); MACN 12750, decimoctava vértebra en vistas lateral (J), craneal (K) y ventral (L). Escala = $10 \mathrm{~mm}$.

Figure 3. Macranhinga paranensis. CICYTTP-PV-A-2-243, seventh vertebra in lateral view (A), cranial (B) and ventral views (C); CICYTTPPV-A-2-242, fifteenth vertebra in lateral (D), cranial (E) and ventral views (F); MLP 88-IX-20-18, seventeenth vertebra in lateral (G), cranial (H) and ventral views (I); MACN 12750, eighteenth vertebra in lateral (J), cranial (K) and ventral views $(\mathbf{L})$. Scale bar $=10 \mathrm{~mm}$. 
$23 \%$ mayor que la de $A$. fraileyi. Presenta una concavidad en la base del processus ventralis, medial y ventral a la facies articularis caudalis, comparable con la decimoctava de $A$. anhinga. El foramen transversarium es más redondeado que en $A$. anhinga. La base de la zygapophysis caudalis presenta rebordes menos agudos y el torus dorsalis más prominente caudalmente que $A$. anhinga. El processus caroticus, en la base del processus costalis, está poco desarrollado y es muy lateral en relación al de $A$. anhinga. El processus ventralis está más desarrollado cranealmente que en $A$. anhinga y A. fraileyi (Campbell, 1996: fig. 1H). Además, comparada con $A$. fraileyi, presenta la porción medial del corpus vertebrae más estrecha, como se observa en $A$. anhinga. Campbell (1996) consideró al ejemplar LACM 135358 como una vértebra decimoctava por la presencia de dos crestas laterales al processus ventralis que convergen cranealmente, delimitando entre ellas una leve concavidad sobre la superficie ventral del cuerpo. Sin embargo, en $A$. anhinga y en el material bajo estudio no se desarrollan dichas crestas en la vértebra decimoctava y, por el contrario, se observan en las vértebras decimonovena y vigésima de la especie actual. La proporción entre el LCV y el AAP no muestra diferencias significativas con $A$. anhinga; pero la relación entre los cuerpos vertebrales y el tamaño de la tróclea III del tarsometatarso exhibe nuevamente un valor menor que en $A$. anhinga y similar al de $A$. fraileyi (Tabla 1$)$.

\section{DISCUSIÓN}

Los elementos apendiculares han sido de suma utilidad para diagnosticar la mayoría especies fósiles de Anhingidae, en tanto que los elementos del esqueleto axial son poco conocidos. En lo que respecta a las Anhingidae de América del Sur se han descrito vértebras referidas a cinco especies: dos cervicales referidas a Anhinga fraileyi (Campbell, 1996), cuatro cervicales a Macranhinga ranzii (Alvarenga \& Guilherme, 2003), una cervical de Liptornis hesternus (Ameghino, 1895), una cervical posiblemente de Anhinga grandis (Rasmussen y Kay, 1992) y cuatro torácicas y una caudal pertenecientes a Meganhinga chilensis (Alvarenga, 1995).

Las proporciones entre los elementos del esqueleto axial y la cintura pélvica en Anhinga anhinga y Macranhinga paranensis son similares (Tabla 1), hecho que apoya la atribución sistemática de los fósiles aquí estudiados a M. paranensis. Dicha similitud interespecífica (entre $A$. anhinga y $M$. paranensis) es esperable, ya que los elementos del esqueleto axial y la cintura pélvica se encuentran estrechamente relacionados través del sinsacro.

Estudios sobre modularidad han determinado que los elementos de la columna vertebral pueden actuar en aves como estructuras accesorias de los demás módulos locomotores (Gatesy \& Dial, 1996), como en este caso la pelvis. Esto permite realizar las siguientes consideraciones.

Al calcular las proporciones entre las vértebras y el tarsometatarso en Anhinga anhinga y Macranhinga paranensis, se obtuvieron pequeñas diferencias (Tabla 1). Esto puede ser interpretado como variaciones intraespecíficas, ya sea ligadas a la edad o al dimorfismo sexual, o bien como diferencias alométricas entre ambas especies, tal como ocurre en otras aves (Acosta Hospitaleche, 2004).

Sin embargo, el hecho de que en los tres casos los valores de las proporciones calculadas en Macranhinga paranensis hayan resultado menores que en Anhinga anhinga parece indicar que el miembro posterior en la forma fósil habría sido proporcionalmente más grande que en su análogo viviente. Asimismo, valores similares en las proporciones de $M$. paranensis y de $M$. ranzii parecen indicar una homogeneidad estructural entre congéneres, independientemente de sus diferentes tamaños.

\section{Contexto paleoambiental}

La Formación Paraná fue depositada en ambientes aéreos o subaéreos a submareales, representados por barreras arrecifales carbonáticas (Aceñolaza \& Aceñolaza, 2000), en algunos sectores con influencias deltaicas (Iriondo, 1973; del Río, 2000) y bajo condiciones climáticas subtropicales a tropicales húmedas (e.g. Herbst \& Zabert, 1987; Zucol \& Brea, 2000).

Los registros paleobotánicos de la Formación Paraná, revisados recientemente por Brea \& Zucol (2011), provienen de las provincias de Santa Fe y Entre Ríos. Indican la presencia de una selva higrófila desarrollada en un ambiente que bordeaba las planicies inundables del mar (Aceñolaza \& Aceñolaza, 1996; Anzótegui \& Aceñolaza, 2006), junto a zonas de vegetación dulceacuícola asociada a cursos de agua torrentosos y en menor medida formas relacionadas a cuerpos de agua lénticos (Anzótegui, 1990; Zucol \& Brea, 2000). En terrenos altos, no inundables, se habrían desarrollado paleocomunidades de sabanas dominadas por palmeras y gramíneas, y bosques tropicales estacionalmente secos (Anzótegui, 1990; Zucol \& Brea, 2000; Brea et al., 2001; Franco \& Brea, 2008).

$\mathrm{Al}$ abundante y diverso registro de vertebrados de origen marino de la Formación Paraná (Cione et al., 2000), deben sumarse los recientes hallazgos de formas continentales provenientes de los niveles más cuspidales de dicha unidad en la localidad fosilífera La Juanita (Cione et al., 2008; Noriega et al., 2009). Estos últimos serían representativos de un depósito de tormenta desarrollado en un ambiente de playa de aguas someras del "Mar Paranense" surcado por arroyos y ríos de agua dulce que desembocaban en él (Cione et al., 2008; Noriega et al., 2009). En cercanías al lugar de procedencia del material aquí estudiado, los hallazgos de foraminíferos y ostrácodos en la misma unidad, indican la presencia de un mar somero, intercalado con sedimentos posiblemente de origen deltaico (Marengo, 2000).

El contexto paleoambiental en el que se depositó la Formación Paraná, reconstruido en base a información geológica y paleontológica, es coincidente con los ambientes estuariales, manglares, bahías poco profundas, lagunas costeras, lagos alcalinos interiores o inclusive zonas deltaicas como los que pueden frecuentar actualmente las 
anhingas (Orta, 1992). Así, el registro de Macranhinga paranensis resultaría acorde con cualquiera de estos posibles escenarios. En todos los casos habría sido imperativo el desarrollo de una vegetación arbórea cercana, si se asume que los requisitos de nidificación de esta paleoespecie eran similares a los de las formas vivientes.

La Formación Ituzaingó se originó por los depósitos de un paleorío (río "Ituzaingó” o "Paleoparaná", según Herbst, 2000), entrelazado, arenoso, de baja sinuosidad, con una gran planicie aluvial, con pendiente y control estructural sobre la margen izquierda, similar al actual río Paraná (Jalfin, 1988). Los registros paleobotánicos de la Formación Ituzaingó dan cuenta de la existencia de elementos representativos de los bosques mixtos xerófilos, bosques dominados por palmeras, bosques mixtos higrófilos y de una asociación dulceacuícola, las dos últimas muy vinculadas con humedales (Anzótegui \& Lutz, 1987; Franco, 2009, 2010; Franco \& Brea, 2009, 2010; Brea \& Zucol, 2011).

La asociación de vertebrados hallada en el "Conglomerado Osífero" se habría conformado bajo condiciones climáticas más cálidas que las actuales (Cione et al., 2000). Los mamíferos y las aves sugieren la presencia de vegetación arbórea densa en las márgenes del antiguo río Paraná y de áreas más abiertas sobre terrenos ubicados más allá de las riberas (Cione et al., 2000; Noriega \& Agnolin 2008; Noriega, 2010).

La presencia de $M$. paranensis en esta unidad resulta entonces claramente compatible con los ambientes inferidos a partir de los registros previos y con los requerimientos ecológicos de los representantes actuales de la familia.

\section{CONCLUSIONES}

Las comparaciones con la especie viviente Anhinga anhinga y las formas fósiles dan cuenta del tamaño compatible de las vértebras aquí presentadas con el esperable para Macranhinga paranensis. Los parámetros biométricos calculados permiten su asignación a $M$. paranensis, constituyendo el primer registro de vértebras para la especie. Mayores certezas respecto a la morfología de la columna vertebral de $M$. paranensis sólo podrán lograrse a través del hallazgo de nuevos y más completos materiales asociados o articulados.

El hallazgo del CICYTTP-PV-A-2-243 en la Formación Paraná (Mioceno medio-tardío) amplía ligeramente el rango temporal de Macranhinga paranensis, ya que el taxón se reportaba hasta el momento sólo en los niveles basales ("Conglomerado osífero" o "Mesopotamiense", Mioceno tardío) de la suprayacente Formación Ituzaingó.

\section{AGRADECIMIENTOS}

A. Kramarz (MACN) y M. Reguero (MLP) por el préstamo de materiales de las colecciones paleontológicas a su cargo; A. Tubaro y C. Kopuchian (MACN) por el acceso a materiales de comparación; J. Ricle por la realización de las figuras; M. Brea y D. Brandoni por sus comentarios y sugerencias; PIP-CONICET y PICT-2007-392 (ANPCYT) financiaron las investigaciones.

\section{REFERENCIAS}

Aceñolaza, F.G. 1976. Consideraciones bioestratigráficas sobre el Terciario marino de Paraná y alrededores. Acta Geológica Lilloana, 3:91-107.

Aceñolaza, F.G. 2000. La Formación Paraná (Mioceno Medio): estratigrafía, distribución regional y unidades equivalentes. In: F.G. Aceñolaza \& R. Herbst (eds.) El Neógeno de Argentina, INSUGEO, Serie de Correlación Geológica 14, p. 9-28.

Aceñolaza, F.G. 2004. Paleobiogeografia de la región Mesopotámica. In: F.G. Aceñolaza (ed.) Temas de la Biodiversidad del Litoral Fluvial Argentino, INSUGEO, Serie de Correlación Geológica 12, p. 25-30.

Aceñolaza, F.G \& Aceñolaza, G.F. 1996. Improntas foliares de una Lauraceae en la Formación Paraná (Mioceno superior), en Villa Urquiza, Entre Ríos. Ameghiniana, 33:155-159.

Aceñolaza, F.G \& Aceñolaza, G.F. 2000. Trazas fósiles del Terciario marino de Entre Ríos (Formación Paraná, Mioceno Medio), República Argentina. Boletín de la Academia Nacional de Ciencias (Córdoba), 64:209-23.

Acosta Hospitaleche, C. 2004. Los pingüinos (Aves, Sphenisciformes) fósiles de Patagonia. Sistemática, biogeografía y evolución. Universidad Nacional de La Plata, Tesis Doctoral, 321 p.

Alvarenga, H.M.F. 1995. A large and probably flightless Anhinga from the Miocene of Chile. Courier Forschungsinstitut Senckenberg, 181:149-161.

Alvarenga, H.M.F. \& Guilherme, E. 2003. The anhingas (Aves: Pelecaniformes) from the upper tertiary (Miocene-Pliocene) of southwestern amazonia. Journal of Vertebrate Paleontology, 23:614-621.

Anzótegui, L.M. 1990. Estudio Palinológico de la Formación Paraná (Mioceno Superior) "Pozo Josefina", Provincia de Santa Fe, Argentina. II Parte: Paleocomunidades. Facena, 9:75-86.

Anzótegui, L.M. \& Aceñolaza, P.G. 2006. Macroflora en la Formación Paraná (Mioceno Medio), en la provincia de Entre Ríos (Argentina). In: CONGRESO ARGENTINO DE PALEONTOLOGÍA Y BIOSTRATIGRAFÍA, 9, 2006. Resúmenes, Córdoba, UNC, p. 29.

Anzótegui, L.M. \& Lutz, A.I. 1987. Paleocomunidades vegetales del Terciario superior (Formación Ituzaingó) de la Mesopotamia argentina. Revista de la Asociación de Ciencias Naturales del Litoral, 18:131-144.

Areta, J.I.; Noriega, J.I. \& Agnolín, F.L. 2007. A giant darter (Pelecaniformes: Anhingidae) from the Upper Miocene of Argentina and weight calculation of fossil Anhingidae. Neues Jahrbuch für Geologie und Palaöntologie Abhandlungen, 243:343-350. doi:10.1127/0077-7749/2007/0243-0343

Baumel, J.J. \& Witmer, L.M. 1993. Osteology. In: J. Baumel; A. King; J. Breazile; H. Evans and J. Vanden Berge (eds.) Handbook of Avian Anatomy, Publications of the Nuttall Ornithological Club, Harvard University, p. 45-132.

Bidegain, J.C. 1993. Primeros análisis paleomagnéticos en sedimentos del Cenozoico tardío en las márgenes del río Paraná. Revista Asociación Geológica Argentina, 48:247-256.

Bravard, A. 1858. Monografia de los terrenos marinos terciarios de las cercanías del Paraná. Paraná, Imprenta del Registro Oficial, $107 \mathrm{p}$.

Brea, M.; Aceñolaza, P.G. \& Zucol, A.F. 2001. Estudio paleoxilológico en la Formación Paraná, Entre Ríos, Argentina. In: SIMPOSIO ARGENTINO DE PALEOBOTÁNICA Y PALINOLOGÍA, 11. Publicación especial 8, APA, Buenos Aires, p. 10.

Brea, M. \& Zucol, A.F. 2011. The Paraná-Paraguay Basin: Geology and Paleoenvironments. In: Historical Biogeography of 
Neotropical Freshwater Fishes, James S. Albert \& Roberto E. Reis (eds.) University of California Press, p. 69-87.

Campbell Jr., K.E. 1996. A new species of giant anhinga (Aves: Pelecaniformes: Anhingidae) from the upper Miocene (Huayquerian) of Amazonian Peru. Contributions in Science, 460:1-9.

Castellanos, A. 1965. Estudio fisiográfico de la provincia de Corrientes. Publicaciones del Instituto de Fisiografia y Geología de Rosario, 49:1-222.

Cenizo, M.M. \& Agnolin, F.L. 2010. The southernmost records of Anhingidae and a new basal species of Anatidae (Aves) from the lower-middle Miocene of Patagonia, Argentina. Alcheringa, 34:493-514. doi:10.1080/03115511003793504

Charrier, R.; Pinto, L. \& Rodríguez, M.P. 2007. Tectonostratigraphic evolution of the Andean orogen in Chile. In: W. Gibbons \& T. Moreno (eds.) Geology of Chile, Special Publication, The Geological Society, p. 21-114.

Cione, A.L.; Azpelicueta, M.M.; Bond, M.; Carilini, A.A.; Casciotta, J.R.; Cozzuol, M.A.; de la Fuente, M.; Gasparini, Z; Goin, F.J.; Noriega, J.; Scillato-Yané, G.J.; Zoibelson, L.; Tonni, E.P.; Verzi, D. \& Vucetich, M.G. 2000. Miocene vertebrates from Entre Ríos province, Argentine. In: F.G. Aceñolaza \& R. Herbst (eds.) El Neógeno en la Argentina, INSUGEO, Serie de Correlación Geológica 14, p. 191-237.

Cione, A.L.; Mennucci, J.; Perez, L. \& Barla, M.J. 2008. Megascyliorhinus trelewensis (Neoselachi) in the upper Miocene of Paraná, central-eastern Argentina. In: F.G. Aceñolaza (ed.) Temas de la Biodiversidad del Litoral Fluvial Argentino, INSUGEO, Serie de Correlación Geológica 17, p. 41-48.

Cozzuol, M.A. 1993. Mamíferos acuáticos del Mioceno Medio y Tardio de Argentina. Sistemática, evolución y biogeografía. Universidad Nacional de La Plata, Tesis Doctoral, 178 p.

De Alba, E.L. 1953, Geología del alto Paraná en relación con los trabajos de derrocamiento entre Ituzaingó y Posadas. Revista de la Asociación Geológica Argentina, 8:129-161.

Del Río, C.J. 1991. Revisión sistemática de los bivalvos de la Formación Paraná (Mioceno Medio), provincia de Entre Ríos, Argentina. Monografias de la Academia Nacional de Ciencias Exactas, Físicas y Naturales, 7:1-93.

Del Río, C.J. 2000. Malacofauna de las Formaciones Paraná y Puerto Madryn (Mioceno marino, Argentina): su origen, composición y significado bioestratigráfico. In: F.G. Aceñolaza \& R. Herbst (eds.) El Neógeno en la Argentina, INSUGEO, Serie de Correlación Geológica 14, p. 9-27.

Franco, M.J. 2009. Leños fósiles de Anacardiaceae en la Formación Ituzaingó (Plioceno), Toma Vieja, Paraná, Entre Ríos, Argentina. Ameghiniana, 46:587-604.

Franco, M.J. 2010. Soroceaxylon entrerriensis gen. et sp. nov. (Moraceae) de la Formación Ituzaingó (Plioceno-Pleistoceno), Cuenca del río Paraná, Argentina. Revista Mexicana de Ciencias Geológicas, 27:508-519.

Franco, M.J. \& Brea, M. 2008. Leños fósiles de la Formación Paraná (Mioceno Medio), Toma Vieja, Paraná, Entre Ríos, Argentina: registro de bosques estacionales mixtos. Ameghiniana, 45:699-718.

Franco, M.J. \& Brea, M. 2009. Primer registro de leños fósiles de Moraceae y Rutaceae de la Formación Ituzaingó (PliocenoPleistoceno), Toma Vieja, Paraná, Argentina. In: CONGRESO ARGENTINO DE CUATERNARIO Y GEOMORFOLOGÍA, 4/ CONGRESSO DA ASSOCIAÇÃO BRASILEIRA DE ESTUDOS DO QUATERNARIO, 12/ REUNIÓN SOBRE EL CUATERNARIO DE AMÉRICA DEL SUR, 2. Actas, La Plata, p. 639-647.
Franco, M.J. \& Brea, M. 2010. Microlobiusxylon paranaensis gen. et sp. nov. (Fabaceae, Mimosoideae) from the Pliocene-Pleistocene of Ituzaingó formation, Paraná basin, Argentina. Revista Brasileira de Paleontología, 13:103-114. doi:10.4072/rbp.2010.2.03

Frenguelli, J. 1920. Contribución al conocimiento de la Geología de Entre Ríos. Academia Nacional de Ciencias de Córdoba, 14:55-256.

Gatesy, S.M. \& Dial, K.P. 1996. Locomotor modules and the evolution of avian flight. Evolution, 50:331-340.

Herbst, R. 1971. Esquema estratigráfico de la provincia de Corrientes, República Argentina. Revista de la Asociación Geológica Argentina, 26:221-243.

Herbst, R. 2000. La Formación Ituzaingó (Plioceno). Estratigrafía y distribución. In: F.G. Aceñolaza \& R. Herbst (eds.) El Neógeno en la Argentina, INSUGEO, Serie de Correlación Geológica 14, p. 181-243.

Herbst, R. \& Zabert, L.L. 1987. Microfauna de la Formación Paraná (Mioceno Superior) de la cuenca Chaco-Paranaense (Argentina). Facena, 7:165-206.

Hernández, R.M.; Jordan, T.E.; Dalenz Farjat, A.; Echevarría, L.; Idleman, B.D. \& Reynolds, J.H. 2005. Age, distribution, tectonics, and eustatic controls of the Paranense and Carribbean marine transgressions in southern Bolivia and Argentina. Journal of South American Earth Sciences, 19:495-512. doi:10.1016/j. jsames.2005.06.007

Iriondo, M.H. 1973. Análisis ambiental de la Formación Paraná en su área tipo. Boletín de la Asociación Geológica de Córdoba, 2:19-24.

Jalfin, G.A. 1988. Formación Ituzaingó (Plio-Pleistoceno) en Empedrado, provincia de Corrientes: un paleorío arenoso entrelazado tipo Platte. In: REUNIÓN ARGENTINA DE SEDIMENTOLOGÍA, 2, 1988. Actas, Buenos Aires, p. 130-134.

Marengo, H.G. 2000. Rasgos micropaleontológicos de los depósitos de la transgresión Entrerriense-Paranense en la cuenca ChacoParanense y noroeste argentino, República Argentina. In: F.G. Aceñolaza y R. Herbst (eds.). El Neógeno de Argentina, INSUGEO Serie de Correlación Geológica 14, p. 29-45.

Noriega, J.I. 1992. Un nuevo género de Anhingidae (Aves: Pelecaniformes) de la Formación Ituzaingó (Mioceno Superior) de Argentina. Notas del Museo de La Plata, Paleontología, 21:217-223.

Noriega, J.I. 1995. The avifauna from the 'Mesopotamian' (Ituzaingó Formation; Upper Miocene) of Entre Ríos Province, Argentina. Courier Forchungsinstitut Senckenberg, 181:141-148.

Noriega, J.I. 2001. Body mass estimation and locomotion of the Miocene pelecaniform bird Macranhinga. Acta Palaeontologica Polonica, 46:115-128.

Noriega, J.I. 2002. Additional material of Macranhinga paranensis Noriega 1992 (Aves: Pelecaniformes: Anhingidae) from the "Mesopotamian" (Ituzaingo Formation: upper Miocene) of Entre Ríos Province, Argentina. In: SYMPOSIUM OF THE SOCIETY OF AVIAN PALEONTOLOGY AND EVOLUTION, 5, 2000. Proceedings, China Press, p. 51-61.

Noriega, J.I. 2010. Las Aves del "Mesopotamiense" (Mioceno tardío) de Entre Ríos, Argentina: paleoambientes y vinculaciones paleobiogeográficas In: CONGRESO ARGENTINO DE PALEONTOLOGÍAYBIOESTRATIGRAFÍA, 10/CONGRESO LATINOAMERICANO DE PALEONTOLOGÍA, 8. Resúmenes, La Plata, p. 104.

Noriega, J.I. \& Agnolín, F.L. 2008. El registro paleontológico de las aves del 'Mesopotamiense' (Formación Ituzaingó; Mioceno tardío-Plioceno) de la provincia de Entre Ríos, Argentina. In: F.G. Aceñolaza (ed.) Temas de la Biodiversidad del Litoral Fluvial Argentino, INSUGEO, Serie Correlación Geológica 17, p. 123-142. 
Noriega, J.I.; Candela, A.M. \& Bonini, R.A. 2009. Primeros registros de vertebrados terrestres en la Formación Paraná (Mioceno medio-tardío), provincia de Entre Ríos, Argentina. Ameghiniana, 46:40R.

Noriega, J.I. \& Piña, C.I. 2004. Nuevo material de Macranhinga paranensis (Aves: Pelecaniformes: Anhingidae) del Mioceno Superior de la Formación Ituzaingó, provincia de Entre Ríos, Argentina. Ameghiniana, 41:115-118.

Orta, J. 1992. Family Anhingidae (darters). In: J. Hoyo, A. Elliott \& J. Sartagal (eds.) Handbook of the Birds of the World, v. 1. Lynx Edicions, Barcelona, p. 354-361.

Pascual, R. \& Odreman Rivas, O. 1971. Evolución de las comunidades de vertebrados del Terciario argentino. Los aspectos paleozoogeográficos y paleoclimáticos relacionados. Ameghiniana, 8:372-412.

Rasmussen, D.T. 1997. Birds. In: R.F. Kay; R.H. Madden; R.L.
Cifelli \& J.J. Flynn (eds.) Vertebrate Paleontology in the Neotropics, Smithsonian Institution Press, p. 171-183.

Rasmussen, D.T. \& Kay, R.F. 1992. A Miocene anhinga from Colombia, and comments on the zoogeographic relationships of South America's Tertiary avifauna. Science Series, Natural History Museum of Los Angeles, 36:225-230.

Rinderknecht, A. \& Noriega, J.I. 2002. Un nuevo género de Anhingidae (Aves: Pelecaniformes) de la Formación San José (Plioceno-Pleistoceno) del Uruguay. Ameghiniana, 39:183-191.

Zucol, A.F. \& Brea, M. 2000. Análisis fitolítico de la Formación Paraná en la Provincia de Entre Ríos. In: F.G. Aceñolaza \& R. Herbst (eds.) El Neógeno de Argentina, INSUGEO, Serie de Correlación Geológica 14, p. 67-76.

Received in July, 2011; accepted in April, 2012. 